\title{
Bronchoscopic Thermal Vapor Ablation: Best Practice Recommendations from an Expert Panel on Endoscopic Lung Volume Reduction
}

\author{
Daniela Gompelmann ${ }^{\mathrm{a}}{ }^{\mathrm{b}} \quad$ Pallav L. Shah ${ }^{\mathrm{c}-\mathrm{e}}$ Arschang Valipour ${ }^{\mathrm{f}}$ \\ Felix J.F. Herth ${ }^{a, b}$ \\ a Pneumology and Critical Care Medicine, Thoraxklinik, University of Heidelberg, Heidelberg, Germany; \\ ${ }^{b}$ Translational Lung Research Center Heidelberg, German Center for Lung Research, Heidelberg, Germany; \\ ${ }^{c}$ Royal Brompton \& Harefield NHS Foundation Trust, London, UK; ${ }^{d}$ Chelsea and Westminster Hospital NHS

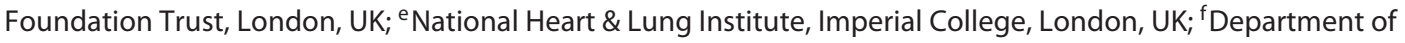 \\ Respiratory and Critical Care Medicine, Ludwig Boltzmann Institute for COPD and Respiratory Epidemiology, \\ Otto-Wagner-Spital, Vienna, Austria
}

\section{Keywords}

Emphysema - Interventional pneumology · Bronchoscopic thermal vapor ablation · InterVapor · Lung volume reduction

\begin{abstract}
Bronchoscopic thermal vapor ablation (BTVA) represents one of the endoscopic lung volume reduction (ELVR) techniques that aims at hyperinflation reduction in patients with advanced emphysema to improve respiratory mechanics. By targeted segmental vapor ablation, an inflammatory response leads to tissue and volume reduction of the most diseased emphysematous segments. So far, BTVA has been demonstrated in several single-arm trials and 1 multinational randomized controlled trial to improve lung function, exercise capacity, and quality of life in patients with upper lobe-predominant emphysema irrespective of the collateral ventilation. In this review, we emphasize the practical aspects of this ELVR method. Patients with upper lobe-predominant emphysema, forced expiratory volume in $1 \mathrm{sec}-$ ond $\left(\mathrm{FEV}_{1}\right)$ between 20 and $45 \%$ of predicted, residual volume (RV) $>175 \%$ of predicted, and carbon monoxide
\end{abstract}

diffusing capacity (DLCO) $\geq 20 \%$ of predicted can be considered for BTVA treatment. Prior to the procedure, a special software assists in identifying the target segments with the highest emphysema index, volume and the highest heterogeneity index to the untreated ipsilateral lung lobes. The procedure may be performed under deep sedation or preferably under general anesthesia. After positioning of the BTVA catheter and occlusion of the target segment by the occlusion balloon, heated water vapor is delivered in a predetermined specified time according to the vapor dose. After the procedure, patients should be strictly monitored to proactively detect symptoms of localized inflammatory reaction that may temporarily worsen the clinical status of the patient and to detect complications. As the data are still very limited, BTVA should be performed within clinical trials or comprehensive registries where the product is commercially available.

(c) 2018 S. Karger AG, Basel

Previous articles in this series: 1 . Shah PL, Herth FJF: Progress in Interventional Pulmonology. Respiration 2018;95:287-288. 2. Bonta PI, Chanez P, Annema JT, Shah PL, Niven R: Bronchial Thermoplasty in Severe Asthma: Best Practice Recommendations from an Expert Panel. Respiration 2018;95:289-300.

\section{KARGER}

(c) 2018 S. Karger AG, Basel

E-Mail karger@karger.com

www.karger.com/res
PD Dr. med. Daniela Gompelmann

Pneumology and Critical Care Medicine, Thoraxklinik

University of Heidelberg, Röntgenstrasse 1

DE-69126 Heidelberg (Germany)

E-Mail daniela.gompelmann@med.uni-heidelberg.de 


\section{Introduction}

Endoscopic lung volume reduction (ELVR) represents an additional therapeutic option for appropriately selected patients with advanced emphysema and severe hyperinflation [1]. Various ELVR techniques with the goal of improving respiratory mechanics are available that differ in their mechanism of action, reversibility, and complication spectrum. The different methods are generally noncompeting procedures, as their application is dependent on the emphysema distribution and degree of collateral ventilation $(\mathrm{CV})$. Bronchoscopic thermal vapor ablation (BTVA; Uptake Medical Technology, Inc., Seattle, WA, USA) is currently intended for bilateral treatment of patients with severe upper lobe-predominant emphysema irrespective of the interlobar CV. By instillation of heated water vapor, a local inflammatory reaction is induced leading to volume and mass reduction of emphysematous segments. It is currently the only technique where no implants are left in situ. Furthermore, the segmental application of heated water vapor allows a targeted therapy of emphysematous destroyed lung segments and thus BTVA has the potential to manage intralobar heterogeneous emphysema. So far, there are several single-arm trials and 1 randomized controlled trial evaluating the safety and efficacy of BTVA $[2,3,6]$. As the data are still very limited, the use of BTVA is still recommended in clinical trials or comprehensive registries where the therapy is commercially available. In Europe, the BTVA registry is actively enrolling patients with upper lobe-predominant emphysema who are being commercially treated with BTVA (NCT03318406). Furthermore, trials in patients with homogeneous and lower lobe-predominant emphysema undergoing BTVA will be initiated in the near future.

This best practice recommendation provides an update on the available scientific data related to BTVA together with an expert recommendation based on clinical experience focused on patient selection, patient preparation, BTVA application, and postprocedural patient management.

\section{Clinical Evidence for BTVA}

In 2009, Snell et al [2] were the first to report on a preliminary series of 11 patients with upper lobe-predominant emphysema, who underwent BTVA with a conservative unilateral lobar dose of $5 \mathrm{cal} / \mathrm{g}$ to the target lung tissue. While the authors were able to confirm feasibility and an acceptable safety profile, efficacy was rather modest, suggesting that a higher dose would be necessary to achieve clinically meaningful outcomes. Subsequently, a lobar vapor dose of $10 \mathrm{cal} / \mathrm{g}$ was chosen to be used in a larger multicenter prospective single-arm study of 44 patients with upper lobe-predominant emphysema (mean $\pm \mathrm{SD}$; forced expiratory volume in 1 second $\left[\mathrm{FEV}_{1}\right] 31.4$ $\pm 7.5 \%$ of predicted; residual volume [RV] $237 \pm 50 \%$ of predicted). The study demonstrated a $48 \%$ target lobar volume reduction at 6 months after treatment, which was accompanied by improvements in lung function (140.8 \pm $26.3 \mathrm{~mL} \mathrm{FEV}{ }_{1}$ improvement, $17 \%$ ), exercise tolerance $(46.5 \pm 15.0 \mathrm{~m}$ in the 6 -minute walking test [6-MWT]), and quality of life $(14.0 \pm 2.4$ point reduction in St. George's Respiratory Questionnaire [SGRQ]) [3]. In a post hoc analysis, the occurrence and severity of serious adverse events increased with the volume of the treated lobe, with an inflection point identified at $1,700 \mathrm{~mL}$ target lobar volume [4]. Given the potential for improving the risk-benefit relationship on the basis of the above, an alternative approach was sought to limit the volume treated in a BTVA session, which has resulted in the design of the STEP-UP study [5]. The STEP-UP study was a randomized, controlled open-label trial using BTVA in a sequential (stepwise) bilateral treatment to target individual segments based on their disease state [6]. Patients included were symptomatic despite optimal medical management and had a baseline mean $\mathrm{FEV}_{1}$ of $33 \%$ with evidence of hyperinflation (mean RV 235\%). The procedure was performed either under conscious sedation or general anesthesia based on institutional standard of practice. The treatment algorithm required vapor ablation of 1 predefined emphysematous segment of an upper lobe during the first session, followed by BTVA of 1 or 2 emphysematous segments in the contralateral upper lobe during a second session performed 12 weeks later. The intention of that approach was to reduce the volume of tissue treated per session and thus the occurrence of serious adverse events, while at the same time increasing the total lung volume treated over the course of the 2 interventions. Results demonstrated a statistically significant mean $14.7 \%$ difference in $\mathrm{FEV}_{1}$ at 6 months after bilateral BTVA compared with the control group and a 9.7-point reduction in SGRQ. About half of the patients in the BTVA group fulfilled the criteria for a minimal clinically important difference in $\mathrm{FEV}_{1}$ and two-thirds for SGRQ at 6 months. Data obtained at the 12-month follow-up visits showed persistent improvements in lung function relative to baseline; however, the magnitude of benefit for $\mathrm{FEV}_{1}$ was slightly less than that documented at 6 months [7]. Im- 
provements in quality of life (SGRQ) in contrast appeared to further increase over time. Importantly, interlobar fissure integrity, an independent predictor of response to bronchoscopic volume reduction therapy with one-way valves, did not influence outcomes with BTVA in both above mentioned multicenter studies [8,9]. With regards to the safety profile, patients in the intervention group experienced a higher pneumonia (18 vs. $8 \%$ ) and COPD exacerbation rate ( 24 vs. $4 \%$ ) at 6 months after treatment compared with controls; however, all events were managed with standard medical treatment representing an improvement over previous lobar-based studies. In summary, results from a limited number of clinical studies (including 1 randomized controlled trial) indicate clinically meaningful improvements in major COPD outcome parameters, with an acceptable safety profile when BTVA is performed with a segmental (rather than lobar) sequential approach.

\section{Patient Selection}

As precise patient selection is the key to success for treating emphysema patients with lung volume therapies, the performance of various examinations that provide an appropriate patient profile for BTVA is essential including body plethysmography, carbon monoxide diffusing capacity (DLCO), blood gas analysis, 6-MWT, echocardiography, multi-detector computed tomography (MDCT), and perfusion scintigraphy. In general, ELVR techniques are only considered in patients with advanced emphysema and persistent symptoms despite optimal pharmacotherapy and pulmonary rehabilitation.

\section{Medical History}

Patients who are considered for any ELVR technique have persistent dyspnea (modified Medical Research Council $[\mathrm{mMRC}] \geq 2$ ) despite optimal pharmacotherapy, ongoing regular physical activity, and cessation of smoking. Only patients with stable COPD ( $\leq 3$ COPD-related hospitalizations requiring antibiotics in past 12 months, no COPD-related hospitalization in past 3 months, and do not require daily use of systemic steroids [ $>5 \mathrm{mg}$ prednisolone]) are suitable for BTVA treatment. Acute COPD exacerbation, bacterial infection, significant sputum, or known microbiological colonization (e.g., Pseudomonas aeruginosa) are contraindications for a BTVA procedure. As BTVA induces an inflammatory reaction, patients with immune system disorders or immunosuppressant medication that would pose a significant increased risk for complications following BTVA should not be treated with BTVA. Also, cardiovascular disorders should carefully be reviewed, specifically patients with a history of myocardial infarction in the previous year, hospitalization due to left ventricular failure in the previous 3 years, or with a stroke should be excluded from BTVA treatment. Due to lack of data, patients with alpha- 1 antitrypsin deficiency should only be treated within clinical trials.

\section{Body Plethysmography}

Based on the inclusion criteria of the clinical trials, patients with a $\mathrm{FEV}_{1}<45 \%$ of predicted were considered for BTVA treatment $[2,3,6]$. The lower limit of $\mathrm{FEV}_{1}$ varied between $15 \%[2,3]$ and $20 \%$ of predicted [6] in the different trials; however, according to our present knowledge, a $\mathrm{FEV}_{1} \geq 20 \%$ of predicted is recommended for safety reasons. As BTVA aims at a reduction of hyperinflation, only patients with increased RV are candidates for this therapeutic approach. A RV $\geq 150 \%$ of predicted was defined as an inclusion criterion for patient enrolment in the different trials $[2,3,6]$. However, in keeping with our consensus statements we recommend that only patients with a RV $\geq 175 \%$ of predicted should be considered tor BTVA treatment [1].

\section{Diffusing Capacity}

In order to tolerate the procedure and potential complications, DLCO, a parameter of the pulmonary reserve, should be $\geq 20 \%$ of predicted when considering patients for BTVA treatment $[2,3,6]$.

\section{Blood Gas Analysis}

Patients with severe hypoxemia $\left(\mathrm{pO}_{2}<50 \mathrm{~mm} \mathrm{Hg}\right.$ on room air) and/or hypercapnia $\left(\mathrm{pCO}_{2} \geq 50 \mathrm{~mm} \mathrm{Hg}\right.$ on room air) should not undergo a BTVA procedure [6]. So far, no data exist on the safety of BTVA in patients with stable disease who receive intermittent noninvasive ventilation, so that it is strictly recommended to treat these patients only within clinical trials.

\section{6-Minute Walking Test}

In general, patients with a reduced 6-MWT distance $(<500 \mathrm{~m})$ can be considered for ELVR. Consistent with the inclusion criteria of the different clinical trials of BTVA, patients with a 6 -MWT distance $\leq 140 \mathrm{~m}$ should not be treated with BTVA due to safety concerns.

\section{Echocardiography}

Baseline echocardiography is recommended to evaluate left and right ventricular function and systolic pulmo- 




Fig. 1. YACTA (yet another CT analyzer) analysis shows an upper lobe-predominant emphysema (yellow color presents low attenuation areas less than a threshold of -950 Hounsfield units). Image courtesy of Prof. Dr. med. Heussel, Thoraxklinik at the University of Heidelberg, Heidelberg, Germany.

nary artery pressure (systolic PAP). Thus far, patients with left ventricular ejection fraction $<40 \%$ were excluded from BTVA procedure $[2,3,6]$. Furthermore, patients with echocardiography results that are suggestive of severe pulmonary hypertension (systolic $\mathrm{PAP}>45 \mathrm{~mm} \mathrm{Hg}$ ) should be considered for right heart catheter catherization. Treatment should not be considered in a patient where an elevated right pulmonary artery pressure (systolic PAP $>45 \mathrm{~mm} \mathrm{Hg}$ or mean PAP $>35 \mathrm{~mm} \mathrm{Hg}$ ) is confirmed for safety reasons [1].

\section{Multi-Detector Computed Tomography}

Noncontrast volumetric MDCT scan with a slice thickness of $0.5-1.25 \mathrm{~mm}$ is required to characterize the emphysema severity, emphysema distribution, and interlobar fissure integrity [1]. So far, efficacy of BTVA has only been confirmed in patients with upper lobe-predominant emphysema, therefore the treatment of patients with homogeneous or lower lobe-predominant emphysema should only be performed within prospective clinical trials. Different CT processing software are available that support the evaluation of emphysematous lung disease in a more quantitative systematic approach, e.g., lobar volumes or lobar emphysema index (Fig. 1). When considering patients for BTVA treatment, a dedicated software - the InterVapor Personalized Procedure Program (IP3) is used. Based on the MDCT data of the patient, a measure of disease severity (tissue-to-air ratio [TAR] or \% -950 Hounsfield units) of each upper lobe segment and of the lower lobes is calculated in order to identify the targeted segment. Only patients with a heterogeneous emphysema with upper lobe predominance (heterogeneity index $[\mathrm{HI}] \geq 1.2$ ) and less diseased lower lobes (TAR $\geq 11 \%$ or $>40 \%$ of areas $>-950$ Hounsfield units) are candidates for BTVA treatment. For each patient who undergoes BTVA, an IP3 data file with 3D reconstruction images of the patient's airways is created demonstrating the anatomy to simplify the procedure. The system also provides possible target locations for treatment and the dose of calorific energy required. As the efficacy of BTVA is independent of interlobar CV, BTVA can be used irrespective of fissure integrity $[8,9]$. Nevertheless, for each patient who is considered for any ELVR treatment, an assessment of interlobar CV is crucial as the degree of CV influences the choice of the ELVR method. Thereby, CV assessment can be performed by CT fissure analysis or invasive catheter-based Chartis ${ }^{\circledR}$ measurement. For patients with advanced emphysema and absent interlobar $\mathrm{CV}$, the reversible valve technique that has already reached the evidence level to be used outside clinical trials should be preferred ahead of the irreversible BTVA - although there is no head-to-head comparison between these two techniques.

MDCT also provides additional information on the lung parenchyma. For example, the presence of a large bulla ( $>1 / 3$ of the lobar volume) is an exclusion criterion for BTVA treatment. Moreover, the CT scan should be carefully reviewed for other pathologies, e.g., pulmonary nodules, infiltration, or bronchiectasis that may suggest the need for further assessment and/or may be an exclusion criterion for BTVA.

\section{Perfusion Scintigraphy}

A perfusion scan is not essential in the assessment for BTVA. However, it is a useful investigation for the evaluation of patients with emphysema being considered for treatment and may be added to the criteria for selecting which segments to reduce.

\section{Key Points}

Patients who fulfil the following minimal selection criteria may be considered as candidates for BTVA treatment:

- Upper lobe-predominant emphysema $(\mathrm{HI} \geq 1.2)$ with a low disease severity of the lower lobe (lower lobe TAR $\geq 11 \%$ or $>40 \%$ of areas $>-950$ Hounsfield units)

- $\mathrm{FEV}_{1}$ between 20 and $45 \%$ of predicted

- $\mathrm{RV} \geq 175 \%$ of predicted

- DLCO $\geq 20 \%$ of predicted

- Clinically stable prior to BTVA treatment

- Persistent symptoms ( $\mathrm{mMRC} \geq 2$ ) despite optimal pharmacotherapy, ongoing regular physical activity, and cessation of smoking 


\begin{tabular}{r|c|c|c|c|c|}
\cline { 2 - 6 } & LUL & LB1 & LB2 & LB3 & LB1+2 \\
\cline { 2 - 6 } TAR: & $10.9 \%$ & $9.9 \%$ & $11.5 \%$ & $12.3 \%$ & $10.3 \%$ \\
\cline { 2 - 6 } Volume, $\mathrm{mL}:$ & 1.5 & 1.6 & 1.4 & 1.3 & 1.6 \\
\cline { 2 - 6 } Percent of lobe: & 2,408 & 1,283 & 340 & 786 & 1,622 \\
\cline { 2 - 6 } & & $53 \%$ & $14 \%$ & $33 \%$ & $67 \%$ \\
\cline { 2 - 6 } Lower lobe TAR: & $16 \%$ & & & & \\
\cline { 2 - 6 } & & & & &
\end{tabular}

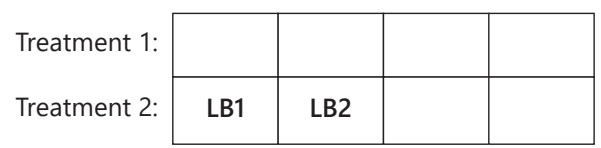

\begin{tabular}{|c|c|c|c|}
\hline RUL & RB1 & RB2 & RB3 \\
\hline $8.9 \%$ & $7.3 \%$ & $9.4 \%$ & $10.9 \%$ \\
\hline 1.5 & 1.8 & 1.4 & 1.2 \\
\hline 3,166 & 1,302 & 1,068 & 797 \\
\hline & $41 \%$ & $34 \%$ & $25 \%$ \\
\hline $13 \%$ & & & \\
\hline
\end{tabular}

\begin{tabular}{|l|l|l|}
\hline RB1 & & \\
\hline & & \\
\hline
\end{tabular}

Fig. 2. Sequential segmental treatment details provided by the InterVapor Personalized Procedure Program (IP3). The target segments are typically those with the highest disease severity, the highest heterogeneity index (HI), and the highest segmental volume. In the right upper lobe, segment 1 (RB1) has the highest disease severity (lowest TAR with $7.3 \%$ ), the highest $\mathrm{HI}$ with 1.8 , and the highest volume with $1,302 \mathrm{~mL}$ and thus presents the target segment.

Key Messages

- Body plethysmography and MDCT scan are the most important examinations to identify candidates for BTVA treatment who are characterized by severe hyperinflation $(\mathrm{RV} \geq 175 \%)$ and upper lobe-predominant emphysema.

- $\mathrm{FEV}_{1}$ and DLCO should be $\geq 20 \%$ due to safety aspects.

\section{Treatment Planning}

BTVA is a bilateral segmental treatment approach in patients with upper lobe-predominant emphysema, whereby the most destroyed lung segment of one upper lobe is the first target followed by the treatment of additional upper lobe emphysematous lung segments 3 months after the first treatment session [6].

Prior to BTVA treatment, the airway target for the procedure as well as the vapor dose that depends on the density and volume of the targeted lung tissue are derived from the patient's preinterventional MDCT by using the IP3 (Fig. 2). For identifying the target segment, the IP3 calculates the disease severity, HI, and volume of each segment of both upper lobes and the lower lobe disease severity. The target segments are those with the highest disease severity, the highest $\mathrm{HI}$, and the highest segmental volume. Based on these results, the IP3 suggests the preferred target segments. Furthermore, the IP3 provides the treatment times or effectively the vapor dose required to treat the specific target area $(8.5 \mathrm{cal} / \mathrm{g}$ lung tissue, derived from the patient's MDCT). For each patient, an IP3 datafile with $3 \mathrm{D}$ reconstruction images of the patient's airways is created and simultaneously displayed during treatment, demonstrating the anatomical structures to simplify and standardize the procedure (Fig. 3).

\section{Key Messages}

- The IP3 that calculates the segmental volumes, the disease severity, and the $\mathrm{HI}$ is used to identify the most emphysematous lung segments (the target segments).

- The goal is to reduce segments with the highest disease severity, the highest HI, and highest segmental volume.

\section{BTVA Treatment}

\section{Patient Management, Sedation, and General Anesthesia}

BTVA treatment can be performed under general anesthesia or deep sedation with local anesthesia - depending on the institutional availability. The goal is to ensure comfort of the patient but it is also essential to minimize coughing or patient movement. Application of the vapor induces a cough and the balloon catheter can be easily displaced applying the energy to an incorrect treatment site. Hence, general anesthesia or at least deep sedation in conjunction with opiates to suppress the cough are neces- 
Fig. 3. Anatomy overview provided by the InterVapor Personalized Procedure Program (IP3). The $3 \mathrm{D}$ reconstruction of the patient's airways is displayed alongside simplifying the identification of the target airway.



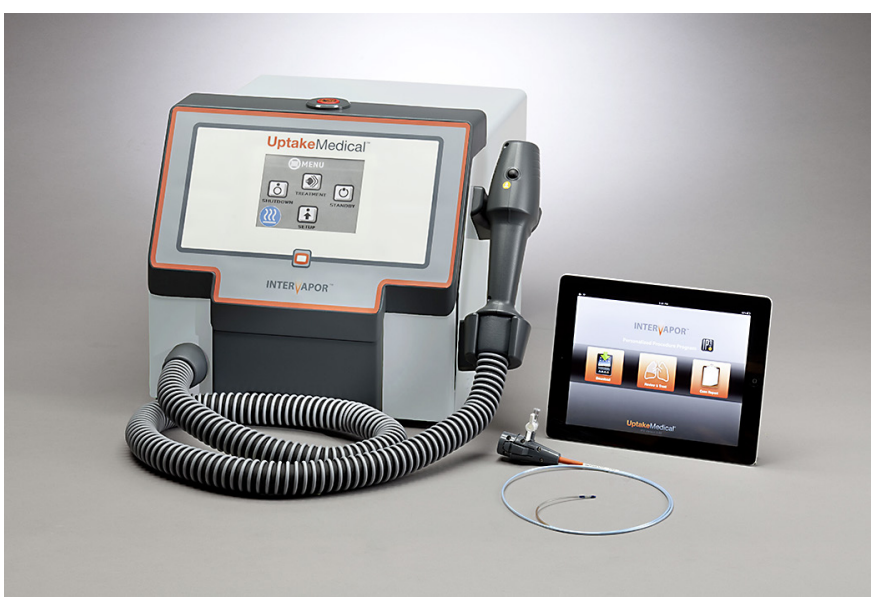

Fig. 4. InterVapor System consisting of a reusable InterVapor Generator and a disposable InterVapor catheter.

sary for the treatment. Strict monitoring of the patient's vital signs is mandatory prior, during, and following the procedure.

If BTVA treatment is performed under general anesthesia, oxygenation and ventilation is maintained via a rigid bronchoscope, endobronchial tube, or laryngeal mask airway that provide access to the airways. If the bronchoscopic procedure is performed under deep sedation, sedative medication should be chosen according to the institution-specific practices. Local anesthetics should be applied topically in the airways, particularly in the target bronchi for BTVA treatment. In case of deep sedation, full readiness to intubate and ventilate is required as these patients with limited pulmonary reserve are prone to develop respiratory and ventilatory failure.

\section{BTVA Treatment}

The InterVapor System consists of an InterVapor Generator, a disposable InterVapor catheter, and the IP3 treatment plan (Fig. 4) [10]. The InterVapor generator is an electronically controlled pressure vessel that generates and delivers precise amounts of the heated water vapor from filled sterile water. The InterVapor catheter is used to deliver the heated water vapor from the generator to the target lung segment. At the distal tip of the catheter, an inflatable silicone balloon is located to occlude the target airway during the treatment, whereas the proximal end of the catheter is connected to the InterVapor Generator.

During bronchoscopy, the airways are inspected for secretion or any pathological findings. Small amounts of secretion can be managed with saline instillation and suction. Samples in the contralateral upper lobe should be obtained and sent for microbiological analysis to guide subsequent antibiotic and or antifungal therapy. In cases where there is marked retention of secretions, the procedure should be abandoned as mucostasis may increase the risk of infectious complications. If no concerning bronchoscopic features are identified, the InterVapor 


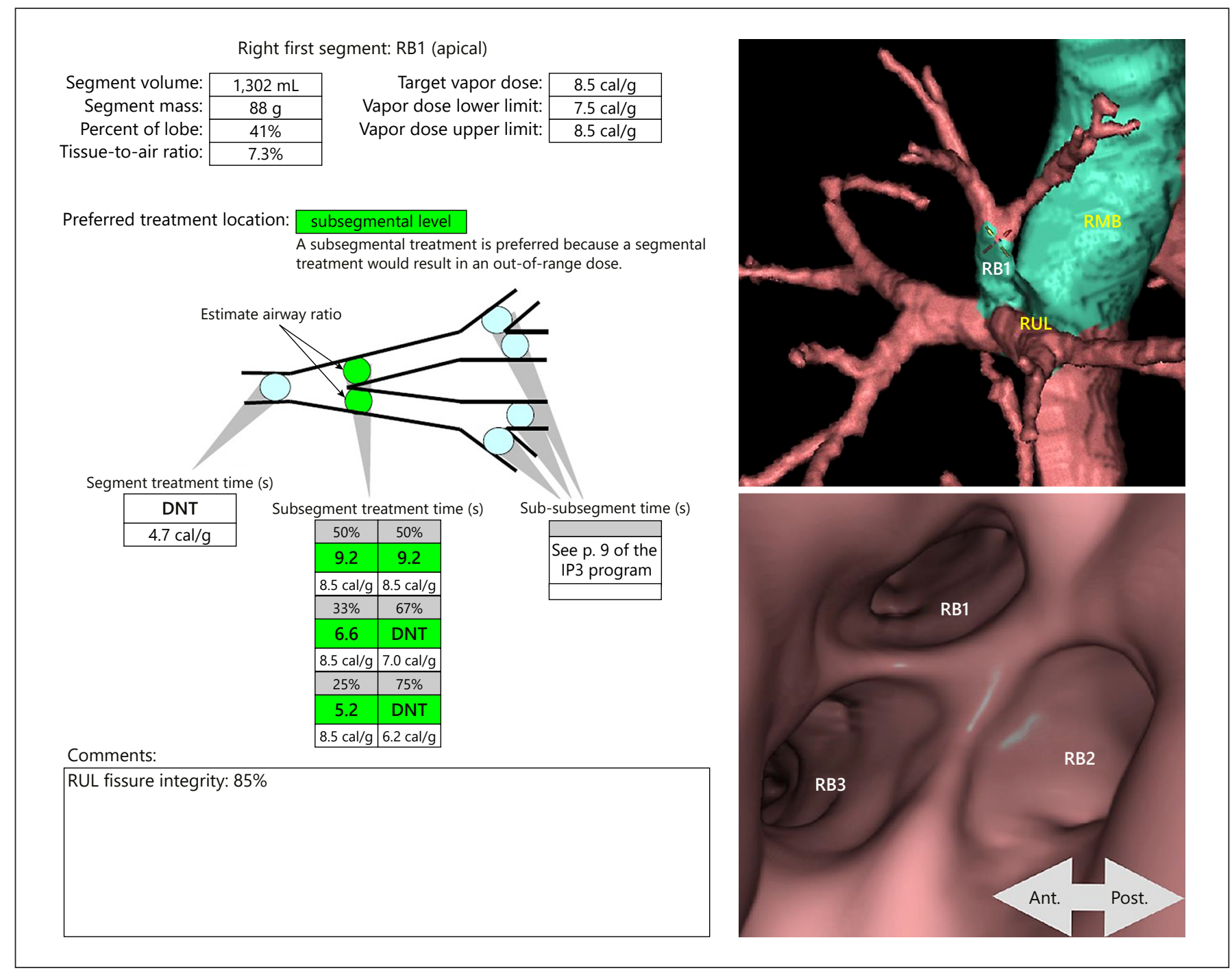

Fig. 5. Treatment plan provided by the InterVapor Personalized Procedure Program (IP3). In this example, the subsegmental level is the preferred treatment location because a segmental treatment would result in an out-ofrange dose. The subsegment treatment time is given by the IP3 and inputted manually by the clinician on the InterVapor Generator screen. DNT, do not treat.

catheter for BTVA treatment is advanced through the instrument channel (currently requires a channel $\geq 2.8$ $\mathrm{mm}$ ) of the flexible bronchoscope into the target bronchi. Thereby, the $3 \mathrm{D}$ reconstruction of the IP3, created prior to the procedure on the basis of the patient's MDCT, is displayed alongside and is used to confirm the airway location for the treatment. Next, the treatment duration provided by the IP3 is reviewed and manually inputted into the Vapor Generator screen (Fig. 5) in order to deliver an $8.5 \mathrm{cal} / \mathrm{g}$ dose. After the airway is sealed by inflating the balloon, the $100^{\circ} \mathrm{C}$ heated water vapor is delivered

Bronchoscopic Thermal Vapor Ablation within 3-10 s. Thereafter, the balloon is deflated and the catheter can be inserted into the next treatment site if required. The time between the two vapor treatments should be at least $3 \mathrm{~min}$. The total procedure time for BTVA is typically less than $15 \mathrm{~min}$.

\section{Postprocedure Management}

After bronchoscopic treatment, the patient should continue to be monitored in the hospital and clinical and vital signs should be recorded at regular intervals. The procedure can be performed in the outpatient setting, but 
where possible the patients should be admitted and monitored overnight. In the first few days after the procedure, patients may report increased cough, sputum, hemoptysis, dyspnea, fever, and chest discomfort. Laboratory tests may show elevated inflammatory markers and chest Xray typically reveals opacification in the treatment area. These clinical, laboratory, and radiological findings are indicative of a noninfectious localized inflammatory reaction in response to BTVA treatment. These patients require increased vigilance in monitoring. Glucocorticosteroids are advised to alleviate symptoms of the local inflammatory reaction (i.e., $0.5 \mathrm{mg} / \mathrm{kg}$ for $2-4$ weeks using a tapering regimen). To avoid a bacterial superinfection during the postprocedure localized inflammatory process, all patients treated with BTVA should receive prophylactic broad-spectrum antibiotics the day of BTVA treatment, which should be continued $\geq 14$ days after the intervention. As complications from the localized inflammatory reaction can occur during the first 2-4 weeks following BTVA, patients should undergo clinical evaluation at 7,14 , and 28 days after treatment. Furthermore, patients must be informed of the need to seek medical help when presenting with any signs or symptoms of inflammatory and/or infectious complications (e.g., increased dyspnea and sputum, fever, hemoptysis).

Key Messages

- BTVA treatment can be performed under general anesthesia or deep sedation. If performed under deep sedation, full readiness to intubate the patient must be given.

- The targeted segment(s) for BTVA treatment is endoscopically identified and confirmed by reviewing the IP3 that provides a $3 \mathrm{D}$ reconstruction of the patient's airways.

- After adequate sealing by the occlusion balloon at the catheter's distal tip in the airway of the targeted segment, heated water vapor is delivered over a dedicated time to deliver the vapor dose.

- Patients must be strictly monitored following the procedure through 4 weeks to proactively detect symptoms of localized inflammatory reaction that may worsen the clinical status of the patient and to detect complications.

\section{Management of Complications}

\section{COPD Exacerbation, Pneumonitis, and Pneumonia}

The most common serious adverse events of BTVA treatment include COPD exacerbation (24\%) and pneumonia or pneumonitis (18\%) [6]. Therefore, we recommend vigilance to proactively detect and treat these complications. As mentioned before, each patient treated with BTVA should receive prophylactic antibiotic therapy to minimize the risk of respiratory exacerbations and bacterial superinfection. Systemic steroids may improve symptoms related to the inflammatory process. In the first weeks following the BTVA procedure, it may be difficult to distinguish between a symptomatic local inflammatory reaction (an expected response to BTVA treatment) and bacterial superinfection as the clinical symptoms are similar. Therefore, symptomatic patients must be monitored very strictly including oxygen saturation, blood gas analysis, laboratory tests, sputum microbiological analysis, and radiological imaging. In case of bacterial superinfection, antimicrobial therapy should be modified according to microbiological lavage findings taken at the time of the procedure. Though not frequent, patients who develop acute respiratory failure may require mechanical ventilation; however, noninvasive ventilation is the first treatment choice. In case of sepsis due to pneumonia, intensive treatment medicine and standard therapeutic strategies according to the guidelines are recommended [11].

\section{Hemoptysis}

Hemoptysis can be observed in 1-3\% of cases following BTVA treatment $[3,6]$. It can be assumed that the hemoptysis is a consequence of the acute inflammatory reaction that is accompanied histologically by alveolar damage, bronchitis, bronchiolitis, and bronchopneumonia [12]. Antibiotic therapy and steroids may reduce the inflammatory process and thus minimize the risk of bleeding. In the clinical trials of BTVA, 1 patient experienced major hemoptysis that was managed by endoscopic balloon tamponade [6].

\section{Pneumothorax}

Pneumothorax is a rare complication following BTVA treatment. In the randomized controlled trials, a rate of $3 \%$ was reported [6]. The single pneumothorax was an incidental finding at 6 months' follow-up and did not require intervention or chest tubes. Despite this, a chest Xray should be performed following the procedure to exclude this complication. In case of pneumothorax, treatment does not differ from the usual pneumothorax management.

\section{Key Messages}

- COPD exacerbations, pneumonitis, and pneumonia are the most common complications following BTVA treatment. Treatment consists of glucocorticosteroids and antimicrobial therapy according to standard care.

- Symptomatic patients must be monitored strictly to proactively detect and treat complications (e.g., bacterial superinfection, lung abscess, sepsis). 


\section{Summary}

BTVA for segmental lung volume reduction in severe emphysema patients typically results in clinically meaningful improvement in pulmonary function, exercise capacity, and quality of life with an acceptable safety profile. However, further clinical experience and data gathered through the BTVA registry and clinical studies will continue to improve patient selection and outcomes of BTVA over time. Therefore, "best practice" will be updated from time to time in order to educate the general pulmonology field on this important ELVR therapy.

\section{Disclosure Statement}

D.G.: Lecture and travel fees from Pulmonx, Olympus, Uptake Medical, Chiesi, Boehringer Ingelheim, Novartis, Astra Zeneca, Mundipharma, Berlin Chemie, and Grifols.

P.L.S.: sponsorship to Imperial College for a bronchoscopy course by Broncus, and Uptake Medical originally reimbursed the Royal Brompton Hospital for clinical trial expenses.

A.V.: Fees for lectures, advisory boards, and/or consultancy from Astra Zeneca, Boehringer Ingelheim, Chiesi, GSK, Pulmonx, Olympus, PneumRx, Novartis, and Uptake Medical.

F.J.F.H.: Fees for lectures and advisory boards from Astra, Almirall, Berlin Chemie, Boehringer, Roche, GSK, Pulmonx, BTG, Olypmus, PneumRx, Boston Scientific, Medupdate, Grifols, CSL Behring, Omniamed, Lilly, Novartis, Teva, Uptake, and Vital Air.

\section{References}

1 Herth FJF, Slebos DJ, Criner GJ, Shah PL: Endoscopic lung volume reduction: an expert panel recommendation - update 2017. Respiration 2017;94:380-388.

-2 Snell GI, Hopkins P, Westall G, Holsworth L, Carle A, Williams TJ: A feasibility and safety study of bronchoscopic thermal vapor ablation: a novel emphysema therapy. Ann Thorac Surg 2009;88:1993-1998.

-3 Snell G, Herth FJ, Hopkins P, Baker KM, Witt C, Gotfried MH, Valipour A, Wagner M, Stanzel F, Egan JJ, Kesten S, Ernst A: Bronchoscopic thermal vapor ablation therapy in the management of heterogeneous emphysema. Eur Respir J 2012;39:1326-1333.

-4 Gompelmann D, Eberhardt R, Ernst A, Hopkins P, Egan J, Stanzel F, Valipour A, Wagner M, Witt C, Baker KM, Gotfried MH, Kesten S, Snell G, Herth FJ: The localized inflammatory response to bronchoscopic thermal vapor ablation. Respiration 2013;86:324-331.

5 Valipour A, Herth FJ, Eberhardt R, Shah PL, Gupta A, Barry R, Henne E, Bandyopadhyay $S$, Snell G: Design of the randomized, controlled sequential staged treatment of emphysema with upper lobe predominance (STEPUP) study. BMC Pulm Med 2014;14:190.

-6 Herth FJ, Valipour A, Shah PL, Eberhardt R, Grah C, Egan J, Ficker JH, Wagner M, Witt
C, Liebers U, Hopkins P, Gesierich W, Phillips M, Stanzel F, McNulty WH, Petermann C, Snell G, Gompelmann D: Segmental volume reduction using thermal vapour ablation in patients with severe emphysema: 6-month results of the multicenter, parallelgroup, open-label, randomized controlled STEP-UP trial. Lancet Respir Med 2016;4: 185-193.

7 Shah PL, Gompelmann D, Valipour A, McNulty WH, Eberhardt R, Grah C, Egan J, Ficker JH, Wagner M, Witt C, Liebers U, Hopkins P, Gesierich W, Phillips M, Stanzel F, Petermann C, Strange C, Snell G, Herth FJ: Thermal vapour ablation to reduce segmental volume in patients with severe emphysema: STEP-UP 12 month results. Lancet Respir Med 2016;4:e44-e45.

-8 Gompelmann D, Heussel CP, Eberhardt R, Snell G, Hopkins P, Baker K, Witt C, Valipour A, Wagner M, Stanzel F, Egan J, Ernst A, Kesten S, Herth FJ: Efficacy of bronchoscopic thermal vapor ablation and lobar fissure completeness in patients with heterogeneous emphysema. Respiration 2012;83:400-406.

-9 Gompelmann D, Eberhardt R, Schuhmann M, Valipour A, Shah PL, Herth FJ, Kontogianni K: Lung volume reduction with vapor ablation in the presence of incomplete fis- sures: 12 -month results from the STEP-UP randomized controlled study. Respiration 2016;92:397-403.

10 Gompelmann D, Eberhardt R, Herth FJ: Technology update: bronchoscopic thermal vapor ablation for managing severe emphysema. Med Devices (Auckl) 2014;7:335-341.

11 Torres A, Niederman MS, Chastre J, Ewig S, Fernandez-Vandellos P, Hanberger H, Kollef M, Li Bassi G, Luna CM, Martin-Loeches I, Paiva JA, Read RC, Rigau D, Timisit JF, Welte T, Wunderink R: International ERS/ESICM/ ESCMID/ALAT guidelines for the management of hospital-acquired pneumonia and ventilator-associated pneumonia: guidelines for the management of hospital-acquired pneumonia (HAP)/ventilator-associated pneumonia (VAP) of the European Respiratory Society (ERS), European Society of Intensive Care Medicine (ESICM), European Society of Clinical Microbiology and Infectious Diseases (ESCMID) and Asociación Latinoamericana del Tórax (ALAT). Eur Respir J 2017;50:1700582.

12 Emery MJ, Eveland RL, Eveland K, Couetil LL, Hildebrandt J, Swenson ER: Lung volume reduction by bronchoscopic administration of steam. Am J Respir Crit Care Med 2010; 182:1282-1291. 